\title{
All Fiber Components for Multimode SDM Systems
}

\author{
Ian Giles ${ }^{1}$, Asiri Obeysekara ${ }^{2}$, Rongsheng Chen ${ }^{1}$, Dean Giles ${ }^{1}$, Francesco Poletti ${ }^{2}$, David Richardson ${ }^{2}$ \\ 1: Phoenix Photonics Ltd., 16 Capital Business Centre, 22 Carlton Road, CR2 0BS, UK ian.giles@phoenixphotonics .com \\ 2: Optoelectronic Research Centre, University of Southampton, Highfield, Southampton, SO7 1BJ, UK
}

\begin{abstract}
Fiber based mode converters and mode splitters are important elements in the FMF mux./demux. Long Period Gratings (LPGs) have been investigated and results presented together with a potential technique for real time mode monitoring during manufacture.
\end{abstract}

\section{INTRODUCTION}

Few moded fiber (FMF) based SDM transmission systems offer major potential to increase transmission capacity. Key to the optimum performance of any system is the component building blocks. As part of the EU supported MODE-GAP project [1] we are investigating the passive fiber component requirements to optimize mode selection and manipulation.

The requirement for the multiplexer is to convert the $\mathrm{LP}_{01}$ mode to a pure stable in-fiber higher order mode and combine the modes into a single fiber. Fiber and waveguide based muxs have been demonstrated [2] and we present results from work on fiber based LPGs and couplers, that can be extended to higher order moded fibers.

An important factor in mode selective component manufacture is the capacity to measure the individual modes in real time whilst fabricating the component. Methods of measuring the modal content of the fiber have been proposed [3, 4], but none that provide real time monitoring. We describe a method to select the individual modes and monitor them during component fabrication.

\section{FIBER MODE CONVERTERS}

Creating a periodic asymmetric perturbation to a fiber capable of supporting $\mathrm{LP}_{01}$ and $\mathrm{LP}_{11}$ modes will convert power in one mode to the other providing the grating period is equal to the modal beat length i.e. $L_{B}=\lambda\left(n_{e 01}-n_{e 11}\right)$, where $\mathrm{n}_{\mathrm{e} 01}, \mathrm{n}_{\mathrm{e} 11}$ are the effective refractive index of the two modes. Squeezing with a periodic mechanical grating, couples light cyclically between the modes [5].

Using fiber with known modal propagation properties a compact mechanical mode converter was developed. The target was to produce an easy to use, adjustable mode converter with wavelength and coupling ratio tuning. Figure 1 shows a schematic of the mode converter and a photograph of the final version. It consists of a steel plate with a 30 period

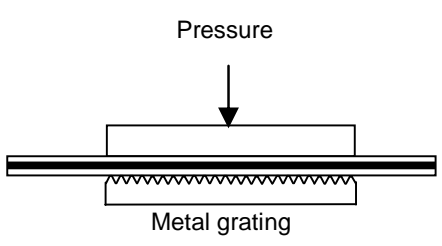

Figure 1 Mechanical prototype tunable LPG

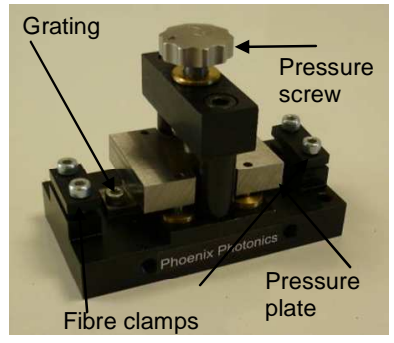

grating cut into it. The plate can be adjusted to vary the angle of the grating relative to the fiber, changing the central coupling wavelength. This length was found to be optimum to provide high coupling with acceptable bandwidth and good stability. The performance of the device was measured using mode strippers on both sides of the grating and coupling into standard SMF giving $30 \mathrm{~dB}$ coupling at $1530 \mathrm{~nm}$ (figure 2). Several of these mode converters were used in the dual mode fiber amplifier experiments [6].

Figure 2 Wavelength response of $\mathrm{LP}_{01}$ throughput for the mechanical LPG

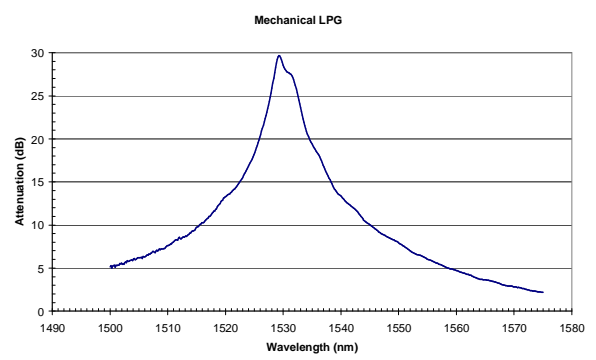

Mechanically tunable gratings offer advantages to provide a good quality $\mathrm{LP}_{11}$ mode at different wavelengths, give a combination of $\mathrm{LP}_{01}$ and $\mathrm{LP}_{11}$ or to switch between the two modes. However fixed gratings offer higher stability and increased flexibility of design. LPGs can be fabricated by several methods and we selected the arc technique [7]. SMF is spliced to both ends of the dual mode fiber and two mode strippers formed on either side of the grating region. The quality of the input $\mathrm{LP}_{01}$ mode is crucial to the fabrication of a good quality grating. Using a broadband ASE source the formation of the grating is monitored on the OSA. The arc is fired and the fiber moved by the modal beat length and then the arc fired again. Figure 3 shows the response of a grating formed by this technique using 34 coupling points, the position of the peak can be modified by changing the stepping distance. 


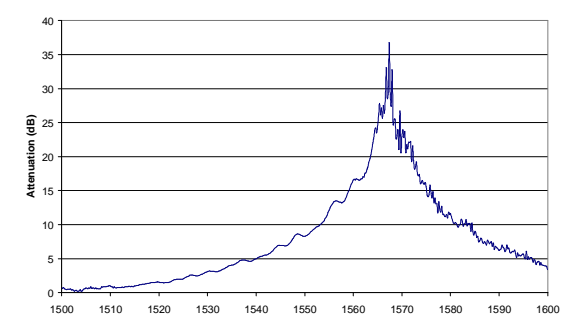

Figure 3 Wavelength response of $\mathrm{LP}_{01}$ throughput for an in-fiber LPG

The gratings were measured using a polarization controlled input such that any input state of polarization could be created and all states scanned over time. It was observed that the output $\mathrm{LP}_{11}$ mode varied between $\mathrm{LP}_{11 \mathrm{a}}$ and $\mathrm{LP}_{11 \mathrm{~b}}$ according to the input state. The variation of spatial mode orientation is created by the beating of the vectorial modes constituting the $\mathrm{LP}_{11}$ mode [8]. Figure 4 shows the variation of the $\mathrm{LP}_{11}$ image for different input polarization states.
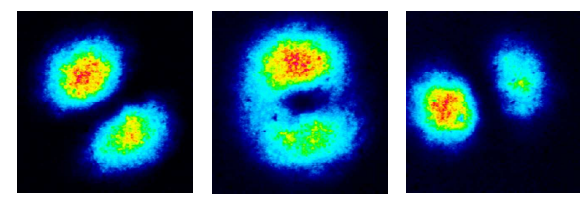

Figure $4 \mathrm{LP}_{11}$ images for changing polarization state into the LPG

\section{MODE SELECTOR}

By tapering the optical fiber the propagating modes can be coupled to radiation modes though a high index overlay material such as a glass prism. Initially used to select modes coupled into an optical fiber [9] the approach has also been used on side polished fibers to measure the effective index of the modes [10]. Access to the propagating modes can be achieved by tapering the fiber, side-polishing to remove cladding or using tilted gratings [11].

Side-polishing an optical fiber over a rotating wheel enables the polishing depth and polish region length to be defined. The finished device has a polished surface that is parallel to the fiber core along the full polished region. Attaching a high index prism to the fiber, couples out the propagating modes to the radiation modes at an angle $(\theta)$ given by $\sin \theta=\left(\mathrm{n}_{\mathrm{p}}{ }^{2}-\mathrm{n}_{\mathrm{e}}{ }^{2}\right)^{1 / 2}$ dependent on the mode effective index $\left(n_{\mathrm{e}}\right)$ and the prism index $\left(n_{p}\right)$. The measured differential divergence angle between $\mathrm{LP}_{01}$ and $\mathrm{LP}_{11}$ modes was $0.29^{0}$ compared to the calculated from fiber data of $0.28^{0}$. The relative divergence is sufficient to enable separation by spatial filtering of each of the radiated modes.

The prism mode selector was used in conjunction with the variable LPG to assess the modal separation. The set-up was a tunable laser input to the mechanical LPG through a single mode fiber, polarization controller and mode stripper. The output from the LPG was spliced into the mode selector. The two radiated output modes were measured on a beam profiler. Figure 5 shows the three output conditions, $\mathrm{LP}_{01}, \mathrm{LP}_{01}+\mathrm{LP}_{11}$
(50/50) and $\mathrm{LP}_{11}$, with limited spatial filtering an isolation of $17 \mathrm{~dB}$ was achieved between the modes.

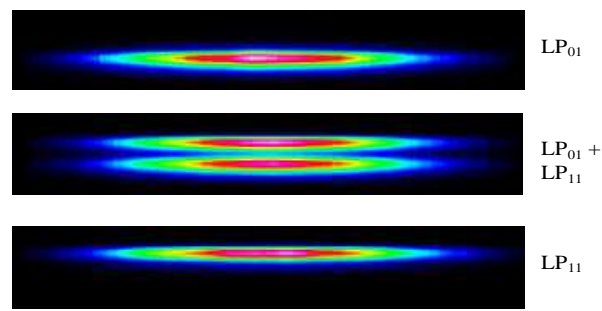

Figure $5 \mathrm{LP}_{01}$ and $\mathrm{LP}_{11}$ modes spatially separated through coupling prism

\section{SUMMARY}

Fiber components are important to provide a low loss, stable multiplex/demultiplex capability in FMF transmission work. The key components are those required to generate the desired modes with a high degree of purity and combine them onto a single transmission fiber with minimum loss and crosstalk. The LPG and fiber couplers are key technologies to realize the necessary components. Methods to monitor the modal content during fabrication are important and the mode selective prism provides the capability to measure the modes independently. Current work has shown the operation of tunable and fixed LPGsand a method of real time modal content monitoring. The technologies under investigation are scalable to higher order FMF.

\section{ACKNOWLEDGMENTS}

This work is supported by EU program FP7-ICT-258033 MODE-GAP. Asiri Obeysekara is supported by a UK EPSRC CASE studentship in conjunction with Phoenix Photonics. Few moded fiber provided by OFS Denmark as part of the MODE-GAP project.

\section{REFERENCES}

[1] EU FP\&-ICT-25033 MODE-GAP www.modegap.eu

[2] N.Hanzawa et al., "Mode-division Multiplexed Transmission with Fiber Mode Couplers," Proceedings of OFC, paper OW1D.4, (2012)

[3] J.W. Nicholson, et al., "Measuring the Modal Content of Large-ModeArea Fibers." Quantum, 15(1), 61-70 (2009)

[4] O.Shapira, et al., "Complete Modal Decomposition for Optical Waveguides." Physical Review Letters, 94(14), 1-4. (2005)

[5] J.N.Blake et al., "Fiber-optic modal coupler using periodic microbending." Optics Letters, Vol. 11, No.3 (1986)

[6] Y.Jung et al., "First demonstration and detailed characterization of a multimode amplifier for space division multiplexed transmission systems.” Optics Express, Vol.19, No26, (2011)

[7] G.Rego et al., "Arc-induced long-period gratings in aluminosilicate glass fibers." Optics Letters, Vol. 30, No. 16 (2005)

[8] Snyder, A. et al.,"Modes of optical waveguides." Energy, 68(3), (1978)

[9] P.S.Szczepanek et al., "Side launch excitation of selected modes in graded-index optical fibers." Applied Optics Vol. 17, No. 20 (1978)

[10] W.V.Sorin et al., "Phase-velocity measaurements using prism output coupling for single- and few-mode optical fibers." Optics Letters, Vol. 11, No. 2 (1986)

[11] Lu Yan et al., "Side-tap modal channel monitor for mode division multiplexed (MDM) systems," Proceedings of OFC, Paper OM3C.2 (2012). 